Revue internationale P.M.E.

Économie et gestion de la petite et moyenne entreprise

\title{
Effets de certaines pratiques de GRH sur la performance de PME manufacturières : vérification de l'approche universaliste
}

\author{
Richard Lacoursière, Bruno Fabi, Josée St-Pierre et Michel Arcand
}

Volume 18, numéro 2, 2005

URI : https://id.erudit.org/iderudit/1008475ar

DOI : https://doi.org/10.7202/1008475ar

Aller au sommaire du numéro

Éditeur(s)

Presses de l’Université du Québec

ISSN

0776-5436 (imprimé)

1918-9699 (numérique)

Découvrir la revue

Citer cet article

Lacoursière, R., Fabi, B., St-Pierre, J. \& Arcand, M. (2005). Effets de certaines pratiques de GRH sur la performance de PME manufacturières : vérification de l'approche universaliste. Revue internationale P.M.E., 18(2), 43-73.

https://doi.org/10.7202/1008475ar
Résumé de l'article

Cette étude a pour but de vérifier si l'on peut constater, en contexte de PME, un effet de certaines pratiques de gestion des ressources humaines (GRH) comparable à celui qui est rapporté par différents chercheurs pour la grande entreprise. Influençant à la fois les conditions de travail, l'organisation du travail, la motivation et le développement des compétences, les pratiques de GRH peuvent constituer une source importante d'avantages concurrentiels pour les entreprises. Les résultats obtenus à partir d'un échantillon de $233 \mathrm{PME}$ manufacturières permettent d'affirmer qu'il existe une relation positive entre le développement des pratiques de GRH et la performance des PME. Des analyses multivariées ont également permis d'attri buer spécifiquement les effets de certaines pratiques sur différentes dimensions de la performance des PME. C'est ainsi que la diffusion d'information apparaît liée au taux de roulement du personnel et au taux de rendement de l'actif ; la formation, à la productivité ; le recrutement et l'évaluation du rendement, à la croissance des ventes. Il apparaît en outre que le rôle significatif qu'exerceraient les pratiques sur la performance varie en fonction de la taille des entreprises. Ces résultats permettent de nuancer les effets des pratiques de GRH en montrant qu'ils peuvent être plus spécifiques que généraux. L’article s'achève sur une discussion intégrant des données d'études antérieures ainsi que des implications de gestion susceptibles d'intéresser des gestionnaires de PME.
Ce document est protégé par la loi sur le droit d'auteur. L'utilisation des services d'Érudit (y compris la reproduction) est assujettie à sa politique d'utilisation que vous pouvez consulter en ligne.

https://apropos.erudit.org/fr/usagers/politique-dutilisation/ 


\title{
Effets de certaines pratiques de GRH sur la performance de PME manufacturières: vérification de l'approche universaliste
}

\author{
Richard LACOURSIÈRE \\ Bruno FABI \\ Josée ST-PIERRE \\ Michel ARCAND \\ Université du Québec à Trois-Rivières
}

\begin{abstract}
LES AUTEURS
RICHARD LACOURSIĖRE, détenteur d'une maîtrise (M.Sc.) en gestion des PME de l'Université du Québec à Trois-Rivières, a œuvré pendant une vingtaine d'années dans le domaine des médias écrits et agi comme consultant dans différents projets de développement organisationnel avant de se joindre à la Chaire de recherche du Canada sur la performance des entreprises. Ses intérêts de recherche concernent particulièrement la gestion des ressources humaines et le rôle que peut exercer cette fonction dans l'atteinte de différents objectifs de performance que poursuivent les organisations. Courriel <Richard_Lacoursiere@uqtr.ca>.

BRUNO $F A B I$ détient un doctorat (Ph.D.) en psychologie industrielle/organisationnelle de l'Université de Montréal. II est professeur titulaire au Département des sciences de la gestion de l'Université du Québec à Trois-Rivières ainsi que chercheur associé à la Chaire de recherche du Canada sur la performance des entreprises. Au cours des 25 dernières années, il a publié de nombreux ouvrages, articles de recherche et professionnels portant notamment sur les pratiques de gestion des ressources humaines, leurs impacts sur la performance organisationnelle et financière, les transformations organisationnelles et le changement stratégique. Son expertise dans ces domaines a également été mise à profit dans le cadre d'interventions en développement international ainsi qu'auprès de diverses organisations privées et publiques de tailles variables et œuvrant dans divers secteurs d'activité. Courriel <Bruno_Fabi@uqtr.ca>.

JoSÉE ST-PIERRE, détentrice d'un doctorat en finance (Ph.D.), dirige le Laboratoire de recherche sur la performance des entreprises (http://www.uqtr.ca/inrpme/larepe). Elle est titulaire de la Chaire J.-Armand-Bombardier sur la gestion du risque et les relations interentreprises, en plus de faire partie du comité d'orientation de l'Institut de recherche sur les PME dont elle est l'un des membres fondateurs. Elle a écrit de nombreux ouvrages sur les difficultés des PME, notamment en matière de financement, sur la performance et le risque. Elle a contribué au développement d'outils spécialisés visant le diagnostic de la performance et du risque des PME, dont PDG et eRisC. Courriel < Josee.St-Pierre@Dr.CGOCable.ca>.

MICHEL ARCAND possède un doctorat ès sciences (gestion) de l'Université de Metz (France). Ses travaux portent principalement sur la mesure et la modélisation des activités de gestion des ressources humaines sur l'efficacité des organisations bancaires et financières. II s'intéresse aussi aux effets de la mondialisation sur la gestion des ressources humaines, à la gestion du changement, ainsi qu'à la méthodologie de recherche. Tout en menant ses activités d'enseignement, le professeur Arcand est intervenu comme conseiller auprès d'entreprises privées et publiques, et ce, autant au Canada qu'en Europe. Courriel $<$ Michel_Arcand@uqtr.ca>.

Remerciements

Les auteurs tiennent à remercier le Programme des chaires de recherche du Canada, la Fondation canadienne pour l'innovation, la Fondation J.-Armand-Bombardier et Développement
\end{abstract} économique Canada de leur soutien financier pour cette recherche. 


\title{
MOTS CLÉS
}

\section{GRH - PME - Formation - Productivité - Performance}

\section{RÉSUMÉ}

Cette étude a pour but de vérifier si l'on peut constater, en contexte de PME, un effet de certaines pratiques de gestion des ressources humaines (GRH) comparable à celui qui est rapporté par différents chercheurs pour la grande entreprise. Influençant à la fois les conditions de travail, l'organisation du travail, la motivation et le développement des compétences, les pratiques de GRH peuvent constituer une source importante d'avantages concurrentiels pour les entreprises. Les résultats obtenus à partir d'un échantillon de 233 PME manufacturières permettent d'affirmer qu'il existe une relation positive entre le développement des pratiques de GRH et la performance des PME. Des analyses multivariées ont également permis d'attribuer spécifiquement les effets de certaines pratiques sur différentes dimensions de la performance des PME. C'est ainsi que la diffusion d'information apparaît liée au taux de roulement du personnel et au taux de rendement de l'actif; la formation, à la productivité; le recrutement et l'évaluation du rendement, à la croissance des ventes. II apparaît en outre que le rôle significatif qu'exerceraient les pratiques sur la performance varie en fonction de la taille des entreprises. Ces résultats permettent de nuancer les effets des pratiques de GRH en montrant qu'ils peuvent être plus spécifiques que généraux. L'article s'achève sur une discussion intégrant des données d'études antérieures ainsi que des implications de gestion susceptibles d'intéresser des gestionnaires de PME.

\begin{abstract}
The objective of this study is to investigate whether one can observe in manufacturing SMEs the effects of some human resource management (HRM) practices comparable to the ones observed by many researchers in large businesses. Affecting working conditions as well as work organization, motivation and skills development, HRM practices can be seen as an important source of competitive advantage for SMEs. The result of sampling 233 SMEs demonstrates that many HRM practices appear to be significantly linked with the performance of an SME. More precisely, our results show that information sharing is linked with turnover and return on assets, that training is linked with productivity and that recruitment and performance appraisal are linked with sales growth. Furthermore, the role played by HRM practices seems to vary in comparison with size of the SMEs. Our results also suggest that the impacts of HRM practices could be specific rather than general. The paper ends with a discussion of our findings and of some managerial implications.
\end{abstract}

\section{RESUMEN}

Este estudio tiene como objetivo averiguar si se puede hacer constar, en contexto de PyMEs, un efecto de ciertas prácticas de gestión de los recursos humanos $(G R H)$ comparable a otro traído por varios investigadores a nivel de la gran 
empresa. Sabiendo que influyan a la vez las condiciones de trabajo, la organización del trabajo, la motivación y el desarrollo de las competencias, las prácticas de GRH pueden constituir una fuente importante de ventajas competitivas para las empresas. Los resultados obtenidos a partir de una muestra de 233 PyMEs manufactureras permiten afirmar que existe una relación positiva entre el desarrollo de las prácticas de GRH y la performancia de las PyMEs. Análisis multivariantes también permitieron atribuir específicamente los efectos de ciertas prácticas sobre diferentes dimensiones de la performancia de las PyMEs. Así es como la difusión de información aparece vinculada al índice de rotación del personal y al índice de rendimiento del activo; la formación, a la productividad; el recrutamiento y la evaluación del rendimiento, al crecimiento de las ventas. Aparece además que el papel significativo que ejercerían las prácticas sobre la performancia varía en función del tamaño de las empresas. Estos resultados permiten matizar los efectos de las prácticas de GRH mostrando que pueden ser más específicos que generales. El artículo acaba con una discusión que integra datos de estudios anteriores asi como implicaciones de gestión suceptibles de interesar a gestores de PyMEs.

\section{ZUSAMMENFASSUNG}

Die vorliegende Studie soll verifizieren, ob in kleinen und mittleren Unternehmen ähnliche Wirkungszusammenhänge von personalpolitischen Aktivitäten bestehen, wie diese von verschiedenen Forschern bei Grossunternehmen festgestellt und beschrieben wurden. Das Personalmanagement nimmt Einfluss auf die Arbeitsbedingungen, die Arbeitsorganisation, die Motivation und die Entwicklung der Kompetenzen. Aus diesen Gründen können Aktivitäten des Personalmanagements entscheidende Wettbewerbsvorteile schaffen. Die Resultate aus einer Umfrage bei 233 kleinen und mittleren Produktionsbetrieben bestätigen, dass positive Beziehungen zwischen den Personalmanagementaktivitäten und der Leistung der KMU bestehen. Multivariate Analysen erlauben zudem eine spezifische Zuteilung von Aktivitäten des Personalmanagements und deren Auswirkungen auf diverse Leistungsdimensionen einer Unternehmung. Es zeigt sich dabei, dass die Informationsverbreitung mit der Personalfluktuationsrate, der Kapitalrendite (die Ausbildung, die Produktivität, Personalrekrutierung, die Erfolgsmessung) und dem Umsatzwachstum verbunden ist. Überdies zeigt sich, dass der Einfluss des Personalmanagements von der Grösse der Unternehmung abhängt. Die Resultate zeigen dementsprechend, dass die Aktivitäten und die damit verbunden Effekte doch nuanciert werden können und nicht nur genereller Natur sind. Der Artikel schliesst mit der Integration bereits bestehender, thematisch ähnlicher Veröffentlichungen und den Auswirkungen, die die Verantwortlichen von KMU interessieren dürften.

\section{Introduction}

La gestion des ressources humaines (GRH) a connu, au cours des dernières années, un essor considérable, devenant un sujet d'études auquel s'intéressent de plus en plus de chercheurs, tant dans le domaine de la grande entreprise que dans celui des petites et moyennes entreprises (PME). Les bouleversements économiques de la dernière décennie ne sont pas étrangers à cet essor. Les changements

Revue internationale P.M.E., vol. 18, n 2, 2005

(C) 2005 - Presses de l'Université du Québec

Édifice Le Delta I, 2875, boul. Laurier, bureau 450, Sainte-Foy, Québec G1V 2M2 - Tél.: (418) 657-4399 - www.puq.ca

Tiré de: Revue internationale P.M.E., vol. 18, n² 2 , sous la direction de Louis Raymond - PME1802N

Tous droits de reproduction, de traduction et d'adaptation réservés 
majeurs auxquels on a assisté à la fin du $\mathrm{Xx}^{\mathrm{e}}$ siècle et l'incertitude croissante qui caractérise l'économie ont eu pour effet de modifier sensiblement les conditions de réussite des entreprises, obligeant plusieurs d'entre elles à réviser leur mode de gestion, de même que leur structure organisationnelle et leur façon d'organiser le travail (Becker et Gerhart, 1996; Schumann, 1998; Tetenbaum, 1998).

L'investissement dans les pratiques de GRH apparaît de plus en plus comme l'une des solutions pouvant permettre aux entreprises d'augmenter leur productivité et d'accroître leur capacité concurrentielle; il s'agirait même d'un des leviers stratégiques les plus malléables dont disposent les dirigeants pour améliorer la rentabilité de leur entreprise (Becker et al., 1997). Même en contexte de PME, où la fonction GRH est généralement moins développée que dans la grande entreprise, le simple fait d'améliorer certaines pratiques pourrait suffire, selon certains auteurs, à conférer un avantage par rapport aux concurrents (Huselid, Jackson et Schuler, 1997; Fabi et Garand, à paraître).

Différentes études ont cherché à établir une relation entre des pratiques de GRH et la performance des entreprises. La grande majorité d'entre elles ont cependant été menées auprès de grandes entreprises, laissant une large place à l'exploration de ce domaine de recherche en contexte de PME. Un appel à l'intensification de la recherche portant sur les pratiques de GRH en PME a d'ailleurs été lancé à maintes reprises au cours des dernières années (Katz et al., 2000 ; Hornsby et Kuratko, 2003; Tansky et Heneman, 2003).

\section{Cadre conceptuel}

L'intérêt grandissant à l'égard de la gestion des ressources humaines en tant que facteur de succès des organisations peut s'expliquer par le biais de différents champs de recherche émanant à la fois de l'économie, de la psychologie, de la finance et de la stratégie (Becker et Huselid, 1998). Chacune de ces disciplines a fourni des arguments à l'élaboration d'une perspective «universaliste» ou de «best practices», voulant que les pratiques de GRH exercent un effet positif sur la performance des entreprises chaque fois qu'on les applique (Pfeffer, 1994). Revoyons brièvement l'origine et la teneur de ces arguments.

D'un point de vue économique tout d'abord, la perspective universaliste de la GRH peut s'appuyer sur des arguments provenant de la théorie du capital humain (Schultz, 1961; Becker, 1962). Suivant celle-ci, le niveau de connaissance, d'habileté et de compétence détenu par les employés représente, au même titre que les autres actifs corporatifs, une importante source de valeur pour la firme (Jackson et Schuler, 1995; Ducharme, 1998).

Revue internationale P.M.E., vol. 18, nº 2, 2005

(C) 2005 - Presses de l'Université du Québec

Édifice Le Delta I, 2875, boul. Laurier, bureau 450, Sainte-Foy, Québec G1V 2M2 • Tél.: (418) 657-4399 - www.puq.ca

Tiré de: Revue internationale P.M.E., vol. 18, $\mathrm{n}^{\circ} 2$, sous la direction de Louis Raymond PME1802N

Tous droits de reproduction, de traduction et d'adaptation réservés 
À ce premier argument économique s'ajoutent ceux avancés par la théorie des coûts de transaction et la théorie de l'agence. Selon Williamson (1981), le fait de mettre en place un système administratif pour encadrer les transactions s'effectuant à l'interne ne suffit pas à garantir leur efficience. Il faut, en plus, examiner les caractéristiques du capital humain nécessaire à la réalisation des transactions en cause et veiller à façonner la relation d'emploi en conséquence. La théorie de l'agence (Jensen et Meckling, 1976; Jones et Wright, 1992), quant à elle, voudrait que les pratiques de GRH constituent une forme de contrat visant à assurer l'arrimage entre les intérêts du principal (le propriétaire de la PME) et ceux de ses agents (les employés) permettant ainsi à l'entreprise de mieux contrôler son risque d'affaires et de réaliser le rendement attendu de ses actifs (D'Arcimoles, 1995 ; Lepak et Snell, 1999; St-Pierre, 1999).

Une approche psychologique de la GRH indique par ailleurs que certaines pratiques peuvent augmenter la motivation et la satisfaction des travailleurs, ce qui permettrait d'influencer leur comportement et d'exercer un impact favorable sur la productivité et la rentabilité des entreprises (Huselid, Jackson et Schuler, 1997; Fabi, Martin et Valois, 1999).

La discipline de la stratégie, enfin, offre une assise théorique importante à l'approche universaliste de la GRH. Comme l'ont démontré Hansen et Wernerfelt (1989), le succès et le développement d'une entreprise ne reposent pas tant sur le choix d'une industrie en croissance ou d'une niche privilégiée que sur la construction d'une organisation humaine efficace au sein de l'industrie choisie. C'était là un argument de poids en faveur de la théorie des ressources, à laquelle Wernerfelt (1984) a largement contribué et qui s'est par la suite enrichie de plusieurs contributions, dont celles de Prahalad et Hamel (1990), Barney (1991) Peteraf (1993), ainsi que de Teece, Pisano et Shuen (1997), pour n'en mentionner que quelques-unes.

S'efforçant de modéliser l'arrimage entre la GRH et la théorie des ressources, Wright, Dunford et Snell (2001) expliquent que les pratiques de GRH (recrutement, formation, participation, évaluation du rendement, récompenses, etc.) contribuent au cœur de métier (core competence) de l'entreprise en agissant à la fois sur son capital intellectuel (intellectual capital), sa gestion des connaissances (knowledge management) et sa capacité de renouvellement (dynamic capability).

La théorie des ressources a donc grandement contribué à l'élaboration du concept de gestion stratégique des ressources humaines (GSRH), autour duquel se sont développées différentes approches que Delery et Doty (1996) ont désignées par les appellations «universaliste», «de contingence» et «de configuration».

Revue internationale P.M.E., vol. 18, nº 2, 2005

(c) 2005 - Presses de l'Université du Québec

Édifice Le Delta I, 2875, boul. Laurier, bureau 450, Sainte-Foy, Québec G1V 2M2 • Tél.: (418) 657-4399 - www.puq.ca

Tiré de: Revue internationale P.M.E., vol. 18, $\mathrm{n}^{\circ} 2$, sous la direction de Louis Raymond • PME1802N

Tous droits de reproduction, de traduction et d'adaptation réservés 
L'approche universaliste, comme nous l'avons déjà mentionné, voudrait que les pratiques les plus reconnues de GRH aient un effet positif chaque fois qu'on les applique. Suivant ce modèle, le simple fait d'appliquer une ou plusieurs pratiques de GRH pourrait influencer directement la performance d'une entreprise. Cette approche a également été désignée sous les appellations de «best practices » et de «one best way» (Delery et Doty, 1996; McMahan, Virick et Wrigth, 1999; Colbert, 2004).

L'approche de contingence propose une nuance à l'approche universaliste en avançant que les pratiques de GRH, pour être efficaces, doivent s'aligner avec d'autres facteurs de contingence de l'organisation et particulièrement avec la stratégie, qui est le principal facteur considéré par la GSRH (Delery et Doty, 1996).

L'approche de configuration (Doty, Glick et Huber, 1993; Meyer, Tsui et Hinings, 1993; Lepak et Snell, 1999), enfin, s'attache à mettre en valeur des ensembles de pratiques plutôt que des pratiques individuelles. Suivant cette dernière approche, les pratiques de GRH auraient un effet synergique (renforcement mutuel) entre elles. Cet effet de synergie entre les pratiques, ajouté à leur interaction avec la stratégie, conduirait à une meilleure performance de l'entreprise.

Bien que toutes ces approches soient utilisées en recherche, l'unanimité n'est pas encore faite quant à savoir laquelle serait la plus appropriée pour analyser l'impact des pratiques de GRH sur la performance des entreprises. Certains soulèvent le caractère quelque peu simpliste de l'approche universaliste, dans la mesure, par exemple, où n'importe quelle pratique individuelle de GRH est facilement imitable et ne peut, de ce fait, fournir un avantage compétitif que pour un court laps de temps, soit jusqu'à ce qu'un compétiteur puisse l'imiter (Barney et Wright, 1998). En ce qui concerne les approches de contingence et de configuration, plusieurs auteurs soulignent qu'il peut se révéler très difficile, voire utopique, de vouloir circonscrire des ensembles ou configurations génériques de pratiques pouvant convenir à tel ou tel type de stratégie (Purcell, 1999; Colbert, 2004). Une autre difficulté que présentent ces approches réside dans la capacité des gestionnaires à effectuer les bons choix de pratiques lorsque vient le temps d'élaborer un système de GRH «sur mesure» pour leur entreprise. À cet égard, Becker et al. (1997) soulignent que les gestionnaires des ressources humaines doivent avoir la capacité de comprendre non seulement les systèmes, mais aussi les relations de cause à effet qui les sous-tendent. Or, il semble que si la plupart des gestionnaires sont habiles à exercer les activités traditionnelles de GRH, ils se montrent beaucoup moins habiles lorsqu'il s'agit de traduire les buts et objectifs de l'entreprise en activités à mettre en œuvre pour les réaliser (Huselid, Jackson et Schuler, 1997). Ce pourrait être justement le cas dans les PME, où les pratiques de GRH sont peu développées (Hornsby et Kuratko, 1990, 2003 ; Marlow et Patton, 1993; Kerr et McDougall, 1999; Fabi et Garand, à paraître) et où l'on 
manque globalement d'expertise dans les méthodes et les techniques (d'Amboise et Garand, 1995; Lacoursière, 2002, p. 153). On comprendra que l'absence d'une telle expertise puisse limiter passablement les bénéfices potentiels attribuables à certaines pratiques (Way et Thacker, 2004). Certains chercheurs sont même d'avis que la mise en place de pratiques de GRH dans les PME consiste souvent en une simple réplique des pratiques appliquées dans d'autres entreprises: «Il semble que la connaissance qu'ont les dirigeants de PME des pratiques de GRH consiste en un mélange d'expérience individuelle, d'informations provenant de leur réseau, d'étalonnage et d'improvisation» (traduction libre), affirment à cet égard Katz et al. (2000).

Comme on peut le voir, chaque approche comporte des limites. Qui plus est, un nombre encore très restreint d'études empiriques ont été réalisées en contexte de PME en vue de valider l'une ou l'autre. Pour notre part, compte tenu des informations relativement peu nuancées (quant à la façon d'appliquer les pratiques de GRH) dont nous disposions dans la base de données utilisée pour la présente étude, nous avons opté pour une vérification de l'approche universaliste. Bien que cette approche comporte des limitations évidentes, nous sommes d'avis, tout comme Colbert (2004), qu'elle fournit néanmoins des informations précieuses sur les pratiques de GRH appliquées dans les entreprises.

\subsection{Recension des études empiriques}

L'approche universaliste de la GRH a été validée à travers bon nombre d'études empiriques, lesquelles ont été menées le plus souvent sur des échantillons ne comportant que des grandes entreprises. À notre connaissance, seulement trois études cherchant à mesurer l'impact d'ensembles diversifiés de pratiques de GRH sur la performance des entreprises ont été réalisées en contexte spécifique de PME, soit une étude de Liouville et Bayad (1995), une autre de Arcand, Bayad et Fabi (2002) et une troisième de Way (2002). Deux autres études (Guzzo, Jette et Katzell, 1985; Patterson et al., 1998) visant à mesurer l'impact d'ensembles diversifiés de pratiques de GRH sur la performance méritent qu'on s'y arrête, puisqu'elles ont porté sur des échantillons regroupant à la fois des PME et des grandes entreprises.

L'étude de Liouville et Bayad (1995), réalisée auprès de 271 PME manufacturières françaises, a permis d'examiner les liens de causalité entre les pratiques de gestion des ressources humaines et les performances sociales, organisationnelles et économiques des PME. Après avoir réparti les entreprises en cinq classes selon le degré d'importance accordé par les dirigeants aux préoccupations de GRH, les auteurs ont analysé et comparé la variance observée dans la performance de chacune de ces classes. Les résultats obtenus confirment que les entreprises ayant une forte orientation «qualitative» de la GRH sont également celles qui affichent la

Revue internationale P.M.E., vol. 18, nº 2, 2005

(C) 2005 - Presses de l'Université du Québec

Édifice Le Delta I, 2875, boul. Laurier, bureau 450, Sainte-Foy, Québec G1V 2M2 • Tél.: (418) 657-4399 - www.puq.ca

Tiré de: Revue internationale P.M.E., vol. 18, n², sous la direction de Louis Raymond • PME1802N

Tous droits de reproduction, de traduction et d'adaptation réservés 
meilleure performance économique. Une orientation qualitative de la GRH signifie, selon ces auteurs, que les ressources humaines sont perçues par l'entreprise comme étant une valeur à optimiser et qu'on ne craint pas d'investir dans un grand nombre de pratiques, dont celles liées à la formation, à la rémunération, à la motivation, à l'information, à la participation, au recrutement et à l'évaluation du rendement. Dans l'étude en question, les chercheurs n'ont cependant considéré que l'effet cumulatif des pratiques, sans distinguer les contributions individuelles de chacune. Poursuivant leur analyse, Liouville et Bayad (1995) ont ensuite cherché à vérifier l'existence de relations particulières entre les divers indicateurs de performance au sein de chacune des classes d'entreprises. Selon ces auteurs, la performance économique (rentabilité, croissance des ventes) serait conditionnée par la performance organisationnelle (productivité, qualité, capacité d'innovation), elle-même conditionnée par la performance sociale (rotation du personnel, absentéisme et satisfaction du personnel).

Dans leur étude menée auprès de 46 succursales d'institutions financières de type coopératif (les Caisses populaires Desjardins), Arcand, Bayad et Fabi (2002) établissent une relation positive entre, d'une part, la présence de certaines pratiques de GRH et, d'autre part, différents indicateurs de performance organisationnelle et financière, dont la satisfaction des employés, l'efficacité des ressources humaines (productivité), les trop-perçus (profits) et le rendement sur investissement (RSI). Plus précisément, les résultats obtenus par ces auteurs font voir que, lorsque entrées simultanément dans une analyse de régression, différentes variables GRH sont associées significativement à chacun des quatre indicateurs de performance, la variance expliquée par le modèle total se situant à $41 \%$ pour la satisfaction, $47 \%$ pour les trop-perçus, $50 \%$ pour les RSI et $37 \%$ pour la performance des RH. Les activités de GRH associées de façon significative à l'un ou l'autre des indicateurs de performance concernaient la sécurité d'emploi, la communication, l'organisation du travail, l'évaluation du rendement et la rémunération. Aucune relation significative n'a été observée pour les pratiques de formation et de dotation. Précisons que l'étude de Arcand, Bayad et Fabi (2002) a été menée dans le secteur des services, où les impacts des pratiques de GRH pourraient être plus facilement perceptibles que dans le secteur manufacturier (Capelli et Crocker-Hefter, 1996; Bowen, Gilliland et Folger, 1999).

Quant à l'étude réalisée par Way (2002) auprès de 446 PME (moins de 100 employés) manufacturières et de services des États-Unis, elle a permis d'établir que les entreprises recourant à des pratiques de GRH généralement associées aux systèmes de haute performance (recrutement, rotation d'emplois, formation, travail en équipe, communication et rémunération) affichaient une diminution du taux de renouvellement de leurs employés, de même qu'une augmentation de la productivité (mesure subjective). Cette amélioration de la productivité ne se vérifiait cependant plus lorsqu'on la mesurait à partir de données objectives 
(productivité par employé). Des analyses complémentaires visant à connaître la contribution individuelle de chacune des pratiques ont permis d'établir que seules les pratiques de rémunération étaient associées de façon significative aux mesures de performance (taux de renouvellement et productivité).

Dans une méta-analyse mesurant l'effet d'une douzaine de pratiques, Guzzo, Jette et Katzell (1985) ont, quant à eux, trouvé une relation positive entre, d'une part, la présence des pratiques de formation et d'évaluation du rendement et, d'autre part, une augmentation de la productivité. Aucun effet significatif n'a été relevé pour les pratiques liées au recrutement et à la rémunération incitative. Selon les auteurs, la présence simultanée de l'ensemble des pratiques étudiées n'a révélé aucun effet de synergie entre elles, leur effet combiné demeurant moindre que la somme des effets individuels.

Enfin, dans une étude longitudinale menée auprès de 67 entreprises manufacturières, Patterson, West, Lawthom et Nickell (1998) ont trouvé que l'ensemble étudié de pratiques de GRH expliquerait dans des proportions respectives de $18 \%$ et $19 \%$ la variance observée en termes de productivité et de rentabilité (profits par employé). Selon ces auteurs, la proportion de variance expliquée par les pratiques de GRH se révèle nettement supérieure à celle expliquée par d'autres pratiques liées à la stratégie, à la technologie, à la recherche et développement (R-D) et à la qualité, dont aucune n'arriverait à expliquer plus de $8 \%$ de variance. Des analyses complémentaires ont permis à ces chercheurs d'établir que l'ensemble de pratiques regroupant le recrutement/sélection, la socialisation, la formation et l'évaluation du rendement serait celui qui explique la plus grande part de la variance observée, tant du point de vue de la productivité que de la rentabilité. Le deuxième ensemble de pratiques expliquant une bonne part de la variance observée aurait trait à l'organisation du travail; cet ensemble inclut la polyvalence, la rotation de postes, la délégation de responsabilités et le travail en équipe. Les pratiques appartenant à un troisième ensemble, soit la communication, les cercles de qualité et la rémunération incitative, ne contribueraient pas, quant à elles, à expliquer une part significative de la variance observée.

Les résultats de ces quelques études font ressortir l'importance de la GRH en contexte de PME. Toutefois, aucune d'elles ne nous renseigne sur l'importance relative des pratiques individuelles de GRH lorsque celles-ci sont appliquées en contexte spécifique de PME manufacturières. En effet, l'étude de Patterson et al. (1998) portait sur un échantillon comportant à la fois des PME et des grandes entreprises; les études de Guzzo, Jette et Katzell (1985), de Arcand, Bayad et Fabi (2002) et de Way (2002) portaient sur des échantillons constitués en tout ou en partie de PME appartenant au secteur des services; quant à l'étude de Liouville et Bayad (1995), elle ne nous renseigne aucunement sur la contribution individuelle

Revue internationale P.M.E., vol. 18, n 2, 2005

(C) 2005 - Presses de l'Université du Québec

Édifice Le Delta I, 2875, boul. Laurier, bureau 450, Sainte-Foy, Québec G1V 2M2 • Tél.: (418) 657-4399 - www.puq.ca

Tiré de: Revue internationale P.M.E., vol. 18, n², sous la direction de Louis Raymond • PME1802N

Tous droits de reproduction, de traduction et d'adaptation réservés 
des pratiques étudiées. D'où la pertinence de notre étude qui entend vérifier les relations entre différentes pratiques de GRH et différents indicateurs de performance dans un échantillon de PME manufacturières.

\subsection{Hypothèse de recherche}

Tant les fondements théoriques de l'approche universaliste que les résultats des études empiriques que nous venons de présenter permettent de croire à l'existence d'un effet significatif des pratiques de GRH sur la performance des entreprises. Dans la présente étude, nous chercherons donc à vérifier les impacts de certaines pratiques de GRH sur la performance des entreprises non pas en fonction de la seule présence de ces pratiques, mais plutôt en fonction de l'intensité (ou étendue) avec laquelle elles sont appliquées. Si l'on admet que la simple présence des pratiques puisse constituer un avantage pour les PME, on peut supposer, a fortiori, que plus on étendra leur application à l'ensemble des catégories de personnel, plus on en retirera d'effets positifs. D'où notre hypothèse:

H1: Il y aura une relation positive entre, d'une part, l'étendue d'application des pratiques de $a$ ) politique de recrutement, $b$ ) descriptions de tâches, $c$ ) évaluation du rendement, $d$ ) diffusion d'information, $e$ ) formation, $f)$ participation aux décisions, $g$ ) partage des profits et, d'autre part, la performance des PME.

\section{Méthode de recherche}

Les informations requises concernant les pratiques de GRH, la stratégie et la performance des entreprises ont été puisées à même la base de données du $\mathrm{PDG}^{\circledR}$, mise en place par le Laboratoire de recherche sur la performance des entreprises (LaRePE). Cette base de données contient des informations provenant de quelque 350 PME manufacturières québécoises comptant entre 6 et 405 employés. On y trouve des informations relatives à plus de 850 variables générales et financières, recueillies à partir d'un questionnaire d'informations confidentielles auquel les répondants devaient joindre les états financiers des cinq derniers exercices. Les entreprises sont contactées directement pour fournir leurs informations générales et financières en échange d'un diagnostic sur leur situation générale. Ce processus de collecte d'information assure une grande fiabilité à la base de données utilisée. Afin de comparer les résultats obtenus concernant l'impact des pratiques de GRH sur différents indicateurs de performance, nous avons éliminé les entreprises pour lesquelles il y avait trop de données manquantes, de façon à assurer la stabilité de l'échantillon à travers les divers modèles analysés. Cette façon de procéder nous a laissé un échantillon de 233 entreprises.

Revue internationale P.M.E., vol. 18, n 2, 2005

(C) 2005 - Presses de l'Université du Québec

Édifice Le Delta I, 2875, boul. Laurier, bureau 450, Sainte-Foy, Québec G1V 2M2 • Tél.: (418) 657-4399 - www.puq.ca

Tiré de: Revue internationale P.M.E., vol. 18, n², sous la direction de Louis Raymond • PME1802N

Tous droits de reproduction, de traduction et d'adaptation réservés 


\subsection{Mesure des variables}

Pour la mesure des variables indépendantes, une étendue de 0 à 5 a été attribuée à chacune des pratiques, la valeur 0 correspondant à l'absence de la pratique et la valeur 5 correspondant à l'étendue maximale pouvant être atteinte. Ainsi, pour les pratiques de recrutement, descriptions de tâches, évaluation du rendement, diffusion d'information et participation aux profits, l'étendue a été mesurée à partir du nombre de catégories de personnel (cadres, employés de bureaux, représentants, contremaîtres, employés de production) touchées par l'application de la pratique. Une étendue de 1 signifie que la pratique n'est appliquée qu'à une seule catégorie de personnel tandis qu'une étendue de 5 signifie que la pratique est appliquée à toutes les catégories de personnel. Dans le cas des entreprises qui comptaient moins de cinq catégories de personnel, nous avons attribué une étendue proportionnelle au nombre de catégories représentées. Ainsi, une entreprise comptant seulement trois catégories de personnel mais qui aurait appliqué la pratique à ces trois catégories se voyait attribuer l'étendue maximale (5).

L'étendue de la pratique de participation aux décisions a été mesurée en tenant compte de la nature des consultations effectuées auprès des employés de production pour des décisions concernant la gestion des opérations $(0$ : informés après les faits; 2 : informés avant les faits; 3 : consultés; 4 : partenaires des décisions ; 5 : mandatés pour prendre des décisions).

Pour la pratique de formation, enfin, l'étendue a été mesurée à partir du budget de formation par employé, que nous avons réparti en cinq classes à partir de la distribution statistique (déciles) obtenue pour cette variable (1:0 \$ - 199\$; $2: 200 \$-319 \$ ; 3: 320 \$-499 \$ ; 4: 500 \$-729 \$ ; 5: 730$ \$ et plus).

La performance des entreprises peut se mesurer à plusieurs niveaux (individus, unités de services, organisation, partenaires, etc.) et se rapporter à des dimensions internes (satisfaction, innovation, productivité, rentabilité, etc.) ou externes (par exemple les impacts sur la communauté ou l'environnement). Il en découle que la mesure de la performance peut faire appel à une multitude d'indicateurs se rattachant aux objectifs stratégiques des entreprises (Kaplan et Norton, 1992; Neely et al., 1997; Kennerley et Neely, 2003).

Dans le cas de la GRH, les chercheurs ont retenu différents indicateurs reliés tantôt de près, tantôt de loin, aux activités de cette fonction. Parmi les indicateurs de performance proximaux, c'est-à-dire pouvant être rattachés plus directement aux pratiques de GRH, mentionnons la satisfaction, l'absentéisme et le taux de renouvellement des employés (Guzzo, Jette et Katzell, 1985; Liouville et Bayad, 1995; Arcand, Bayad et Fabi, 2002); parmi les indicateurs les plus lointains (ou

Revue internationale P.M.E., vol. 18, nº 2, 2005

(C) 2005 - Presses de l'Université du Québec

Édifice Le Delta I, 2875, boul. Laurier, bureau 450, Sainte-Foy, Québec G1V 2M2 - Tél.: (418) 657-4399 - www.puq.ca

Tiré de: Revue internationale P.M.E., vol. 18, n², sous la direction de Louis Raymond • PME1802N

Tous droits de reproduction, de traduction et d'adaptation réservés 
distaux), mentionnons le taux de rendement de l'actif, le taux de rendement des fonds propres, de même que le prix des actions (Huselid, 1995; Delery et Doty, 1996; Bryson, 1999; Welbourne et Cyr, 1999); des indicateurs tels que la productivité, la flexibilité, l'innovation et la satisfaction des clients, que l'on qualifie aussi d'intermédiaires, se situent pour leur part entre les plus rapprochés et les plus lointains (Delaney et Huselid, 1996; Ahmad et Schroeder, 2003; Way, 2002).

Pour les fins de la présente étude, nous avons retenu quatre indicateurs de performance, soit un indicateur proximal (le taux de roulement du personnel), deux indicateurs intermédiaires (la productivité et le taux de croissance des ventes) et un indicateur distal (le taux de rendement de l'actif).

Le taux de renouvellement du personnel a été obtenu en divisant le nombre de départs volontaires survenus durant une année par le nombre total d'employés au cours de cette même année. Compte tenu du fait que les employés de production constituent plus de $70 \%$ du personnel des entreprises manufacturières, c'est le ratio de productivité de cette catégorie d'employés qui nous intéresse davantage. Ce ratio a été obtenu en divisant le montant du bénéfice brut par le nombre d'employés de production (employés et contremaîtres). La croissance des ventes a été mesurée en calculant la croissance annuelle moyenne des ventes au cours des cinq dernières années. Enfin, le taux de rendement de l'actif a été obtenu en calculant le ratio du bénéfice avant intérêts et impôts à l'actif total.

Afin de tenir compte des spécificités des PME et de leur hétérogénéité, nous avons ajouté à notre étude deux variables de contrôle, soit l'âge des entreprises et leur taille (nombre d'employés). Nous croyons que la présence de certaines pratiques de même que l'étendue de leur application peuvent varier en fonction des besoins des entreprises, ceux-ci pouvant être mesurés par leur âge ou leur taille. Ainsi, les jeunes PME manufacturières seraient plus préoccupées par l'organisation de leur production et la recherche d'un marché stable, alors que les plus petites PME n'auraient pas une structure suffisamment complexe pour justifier la présence d'un ensemble élaboré de pratiques.

\section{Résultats}

Les données obtenues nous permettent de décrire les principales caractéristiques des PME de notre échantillon par rapport aux différentes variables retenues dans notre modèle de recherche, tout comme elles nous ont permis d'effectuer des analyses de corrélation, puis des analyses multivariées visant à faire ressortir les variables permettant le mieux de prévoir la performance des entreprises. 


\subsection{Résultats descriptifs}

L'analyse de notre échantillon de 233 PME manufacturières permet d'observer que l'âge des entreprises varie entre 3 et 122 ans, la moyenne étant de 24 ans et la médiane se situant à 20 ans. La taille des entreprises oscille entre 20 et 405 employés, la moyenne étant de 69 et la médiane, de 48 employés. En termes économiques, les PME de notre échantillon réalisent un chiffre d'affaires moyen de 8,6 millions de dollars, la médiane s'établissant à 5,4 millions de dollars. Du point de vue de la gestion des ressources humaines, 51\% des PME de notre échantillon disent avoir un responsable désigné pour la fonction GRH et ce responsable est considéré par l'entrepreneur comme étant un employé clé dans $73 \%$ des cas.

Concernant les indicateurs de performance retenus comme variables dépendantes dans nos différents modèles, le tableau 1 montre que le taux de renouvellement du personnel se situe en moyenne à 15,3\% au sein des PME de notre échantillon et que la productivité par employé de production se chiffre en moyenne à $40830 \$$. La croissance annuelle moyenne des ventes au cours des cinq dernières années a été de $34,5 \%$ et le taux de rendement de l'actif dépasse légèrement les $13 \%$.

TABLEAU 1

Situation des PME relativement aux indicateurs de performance $(n=233)$

\begin{tabular}{lll}
\hline & Moyenne & Médiane \\
\hline Taux de renouvellement du personnel & $15,3 \%$ & $8,9 \%$ \\
Productivité du personnel de production & $40830 \$$ & $29906 \$$ \\
Croissance annuelle moyenne des ventes (5 ans) & $34,5 \%$ & $15,6 \%$ \\
Taux de rendement de l'actif & $13,1 \%$ & $11,1 \%$ \\
\hline
\end{tabular}

En ce qui concerne le comportement des PME par rapport aux variables indépendantes, on peut observer au tableau 2, d'une part, que ce ne sont pas toutes les PME qui appliquent les différentes pratiques de GRH et, d'autre part, que l'intensité d'application de ces pratiques varie considérablement ${ }^{1}$. C'est ainsi que seulement 44,4\% des PME disposent d'une politique de recrutement; les PME appliquant cette pratique, toutefois, le font pour plus de quatre catégories d'employés sur cinq $(4,26 / 5,00)$. Un plus grand nombre d'entreprises $(82,3 \%)$ disposent de descriptions de tâches, mais celles-ci visent un moins grand nombre d'employés que la politique de recrutement $(3,70 / 5,00)$. L'évaluation du rendement apparaît elle aussi largement

1. La dernière colonne du tableau donne l'étendue moyenne des pratiques, soit le nombre moyen de catégories d'employés visé. Celui-ci va de un à cinq sauf pour les pratiques concernant la diffusion des informations, qui a une étendue maximale de quatre.

Revue internationale P.M.E., vol. 18, nº 2, 2005

(C) 2005 - Presses de l'Université du Québec

Édifice Le Delta I, 2875, boul. Laurier, bureau 450, Sainte-Foy, Québec G1V 2M2 • Tél.: (418) 657-4399 - www.puq.ca

Tiré de: Revue internationale P.M.E., vol. 18, n², sous la direction de Louis Raymond • PME1802N

Tous droits de reproduction, de traduction et d'adaptation réservés 
répandue $(72,4 \%)$ dans les PME de notre échantillon, mais elle vise encore moins de catégories de personnel que les deux pratiques précédentes $(3,38 / 5,00)$. L'utilisation d'une politique de rémunération incitative, soit le partage des profits, se révèle être la pratique la moins répandue (37\% des PME) et son application est peu uniforme dans les entreprises. On reconnait ici le caractère individuel de la PME, où les décisions émanent souvent du seul propriétaire-dirigeant. La mise en place d'une politique de partage des profits exige la divulgation de certaines informations financières aux employés, une pratique peu utilisée s'il faut en croire nos résultats: la divulgation des informations financières, de fait, demeure concentrée au niveau de la direction de l'entreprise $(1,90 / 4,00)$. En ce qui concerne la diffusion des autres types d'information, toutes les entreprises appliquent cette pratique, mais avec une intensité plus ou moins forte. C'est ainsi que la mission de l'entreprise et les résultats de productivité font l'objet d'une très large diffusion (plus de trois catégories d'employés), tandis que l'évolution de la concurrence et la situation du marché font l'objet d'une diffusion moins étendue. Du côté de la formation, l'utilisation de cette pratique par toutes les PME s'explique par l'existence d'une loi québécoise obli-

TABLEAU 2

Situation des PME relativement à l'étendue moyenne des pratiques de GRH

\begin{tabular}{|c|c|c|c|c|c|c|c|}
\hline & \multirow{2}{*}{$\begin{array}{l}\text { Pourcentage } \\
\text { d'entreprises } \\
\text { appliquant } \\
\text { la pratique }\end{array}$} & \multicolumn{6}{|c|}{$\begin{array}{l}\text { Distribution des entreprises (\%) } \\
\text { selon l'étendue des pratiques }\end{array}$} \\
\hline & & 1 & 2 & 3 & 4 & 5 & $\begin{array}{l}\text { Étendue } \\
\text { moyenne }\end{array}$ \\
\hline Politique de recrutement & 44,4 & 8,1 & 6,0 & 6,5 & 4,7 & 18,9 & 4,26 \\
\hline Descriptions de tâches & 82,3 & 9,0 & 10,3 & 12,5 & 14,2 & 36,2 & 3,70 \\
\hline Évaluation du rendement & 72,4 & 14,2 & 8,6 & 10,8 & 12,9 & 25,8 & 3,38 \\
\hline Partage des profits & 37,0 & 12,1 & 4,3 & 3,4 & 5,1 & 12,1 & 3,02 \\
\hline Information mission entreprise* & 100 & 9,3 & 12,9 & 11,1 & 66,6 & - & 3,35 \\
\hline $\begin{array}{l}\text { Information résultats de } \\
\text { productivité* }^{*}\end{array}$ & 100 & 1,8 & 16,8 & 30,5 & 52,6 & - & 3,37 \\
\hline Information résultats financiers* & 100 & 40,9 & 40,4 & 6,2 & 12,4 & - & 1,90 \\
\hline $\begin{array}{l}\text { Information évolution } \\
\text { de la concurrence* }\end{array}$ & 100 & 13,4 & 41,5 & 14,3 & 31,8 & - & 2,67 \\
\hline $\begin{array}{l}\text { Information situation } \\
\text { du marché* }\end{array}$ & 100 & 13,5 & 35,9 & 15,7 & 35,0 & - & 2,72 \\
\hline Formation & 100 & 17,3 & 28,0 & 20,4 & 13,3 & 20,9 & 2,92 \\
\hline Participation aux décisions & 88,9 & - & 34,3 & 33,9 & 18,9 & 1,7 & 2,86 \\
\hline
\end{tabular}

* 1: PDG et conseil d'administration ; 2 : directeurs/responsables ; 3 : contremaîtres/chefs d'équipe ; 4: employés 
geant les entreprises à y consacrer un minimum de $1 \%$ de leur masse salariale. On observe cependant que les activités de formation s'adressent en moyenne à moins de trois catégories d'employés sur cinq $(2,92 / 5,00)$. Enfin, la participation aux décisions se pratique dans $88,9 \%$ des PME; notons cependant qu'il s'agit d'une participation plutôt limitée, puisque la majorité des entreprises affichent une étendue de $2(34,3 \%)$ ou de 3 (33,9\%), ce qui correspond à des échanges informatifs ou consultatifs; seulement $18,9 \%$ des PME disent appliquer la pratique jusqu'au point de rendre les employés partenaires des décisions et ces derniers ne sont mandatés pour prendre eux-mêmes les décisions que dans $1,7 \%$ des entreprises.

\subsection{Corrélations}

Notre première série de tests statistiques a consisté en des analyses de corrélation nous donnant un aperçu des relations que présentent nos différentes variables les unes avec les autres. Concernant les variables de contrôle tout d'abord, la matrice des corrélations (tableau 3) permet de constater que l'âge des entreprises présente des relations significatives avec trois des quatre indicateurs de performance retenus pour notre étude, soit la productivité par employé de production $(r=0,16 ; \mathrm{p} \leq 0,01)$, la croissance moyenne des ventes $(r=-0,50 ; \mathrm{p} \leq 0,01)$ et le taux de rendement de l'actif $(r=-0,14 ; \mathrm{p} \leq 0,05)$, tandis que le taux de roulement présente un coefficient de corrélation se rapprochant du seuil de signification à $0,1(r=-0,11)$. En ce qui concerne la taille des entreprises, elle présente une corrélation négative avec le taux de roulement du personnel $(r=-0,14$; $\mathrm{p} \leq 0,05)$. Ce dernier résultat pourrait confirmer les difficultés qu'ont les plus petites entreprises à conserver leur personnel, d'où l'importance de mettre en place certaines pratiques de GRH favorisant la rétention.

Toujours au regard des variables de contrôle, on relève la présence de relations significatives avec plusieurs variables indépendantes. L'âge des entreprises, tout d'abord, est associé négativement avec l'étendue de la diffusion d'information portant sur les résultats de productivité $(r=-0,20 ; \mathrm{p} \leq 0,01)$. Quant à la taille, elle présente des relations significatives et positives avec l'étendue des pratiques de recrutement $(r=0,23 ; \mathrm{p} \leq 0,01)$, évaluation du rendement $(r=0,18 ; \mathrm{p} \leq 0,01)$, diffusion d'information relative au marché $(r=0,12 ; \mathrm{p} \leq 0,1)$, formation $(r=0,14$; $\mathrm{p} \leq 0,05)$ et partage des profits $(r=0,12 ; \mathrm{p} \leq 0,1)$, mais une relation négative avec la participation aux décisions $(r=-0,14 ; \mathrm{p} \leq 0,05)$.

Plusieurs corrélations significatives sont par ailleurs observées entre les variables indépendantes et les variables dépendantes. C'est ainsi que trois pratiques de GRH relatives à la diffusion d'information présentent des corrélations significatives avec le taux de roulement du personnel, le signe négatif des coefficients indiquant une diminution de celui-ci. Il s'agit des pratiques de diffusion

Revue internationale P.M.E., vol. 18, nº 2, 2005

(C) 2005 - Presses de l'Université du Québec

Édifice Le Delta I, 2875, boul. Laurier, bureau 450, Sainte-Foy, Québec G1V 2M2 • Tél.: (418) 657-4399 - www.puq.ca

Tiré de: Revue internationale P.M.E., vol. 18, n², sous la direction de Louis Raymond • PME1802N

Tous droits de reproduction, de traduction et d'adaptation réservés 
d'information ayant trait à la mission de l'entreprise $(r=-0,20 ; \mathrm{p} \leq 0,01)$, à l'évolution de la concurrence $(r=-0,18 ; \mathrm{p} \leq 0,01)$ et à la situation du marché $(r=-0,16 ; \mathrm{p} \leq 0,01)$.

Deux autres pratiques de GRH présentent des corrélations significatives avec la productivité du personnel de production. Il s'agit des pratiques de formation $(r=0,26 ; \mathrm{p} \leq 0,01)$ et de participation aux décisions $(r=-0,16 ; \mathrm{p} \leq 0,01)$, cette dernière faisant l'objet d'une relation négative.

Cinq pratiques de GRH présentent par ailleurs des corrélations significatives avec la croissance des ventes des cinq dernières années. Il s'agit des pratiques de descriptions de tâches $(r=0,13 ; \mathrm{p} \leq 0,05)$, recrutement $(r=0,13 ; \mathrm{p} \leq 0,05)$, évaluation du rendement $(r=0,15 ; \mathrm{p} \leq 0,01)$, diffusion des résultats de productivité $(r=0,12 ; \mathrm{p} \leq 0,1)$ et participation aux décisions $(r=0,14 ; \mathrm{p} \leq 0,05)$.

Enfin, deux pratiques présentent des corrélations significatives avec le taux de rendement de l'actif, soit celle des descriptions de tâches $(r=0,12 ; \mathrm{p} \leq 0,1)$ et celle de diffusion d'informations relatives aux états financiers $(r=-0,14$; $\mathrm{p} \leq 0,05)$, cette dernière affichant un signe négatif.

Dans l'ensemble, les résultats des premiers tests supportent notre hypothèse. Les corrélations positives et significatives que présente l'étendue de plusieurs pratiques de GRH avec l'un ou l'autre des indicateurs de performance plaident en faveur de l'approche universaliste voulant que certaines pratiques reconnues de GRH exercent un effet bénéfique sur la performance des entreprises.

\subsection{Régressions multiples}

Afin de vérifier l'effet conjoint des pratiques de GRH sur les indicateurs de performance retenus pour notre étude, nous avons procédé à des analyses multivariées à partir de la technique de régression multiple «Adjusted R-square Best». À partir d'un ensemble donné de variables contenues dans un modèle, ce type de régression ne retient que celles qui permettent d'obtenir le plus fort coefficient d'explication ajusté au niveau de la variable dépendante. L'absence de résultats pour différentes variables dans les cellules du tableau 4 signifie donc que les variables en question n'ont pas été reconnues comme pouvant contribuer à l'explication de la variance observée. De plus, compte tenu de l'hétérogénéité des PME soulevée précédemment et afin d'obtenir une explication la plus nuancée possible des effets des pratiques de GRH sur la performance des PME, nous avons scindé notre échantillon principal (E1) en deux sous-échantillons, en nous basant sur la médiane du nombre d'employés. Nous supposons ainsi que la taille peut influencer non seulement la mise en place des pratiques, mais également leur effet sur la performance des entreprises. La division de l'échantillon permettra de faire ressortir des comportements différents des plus petites entreprises par 


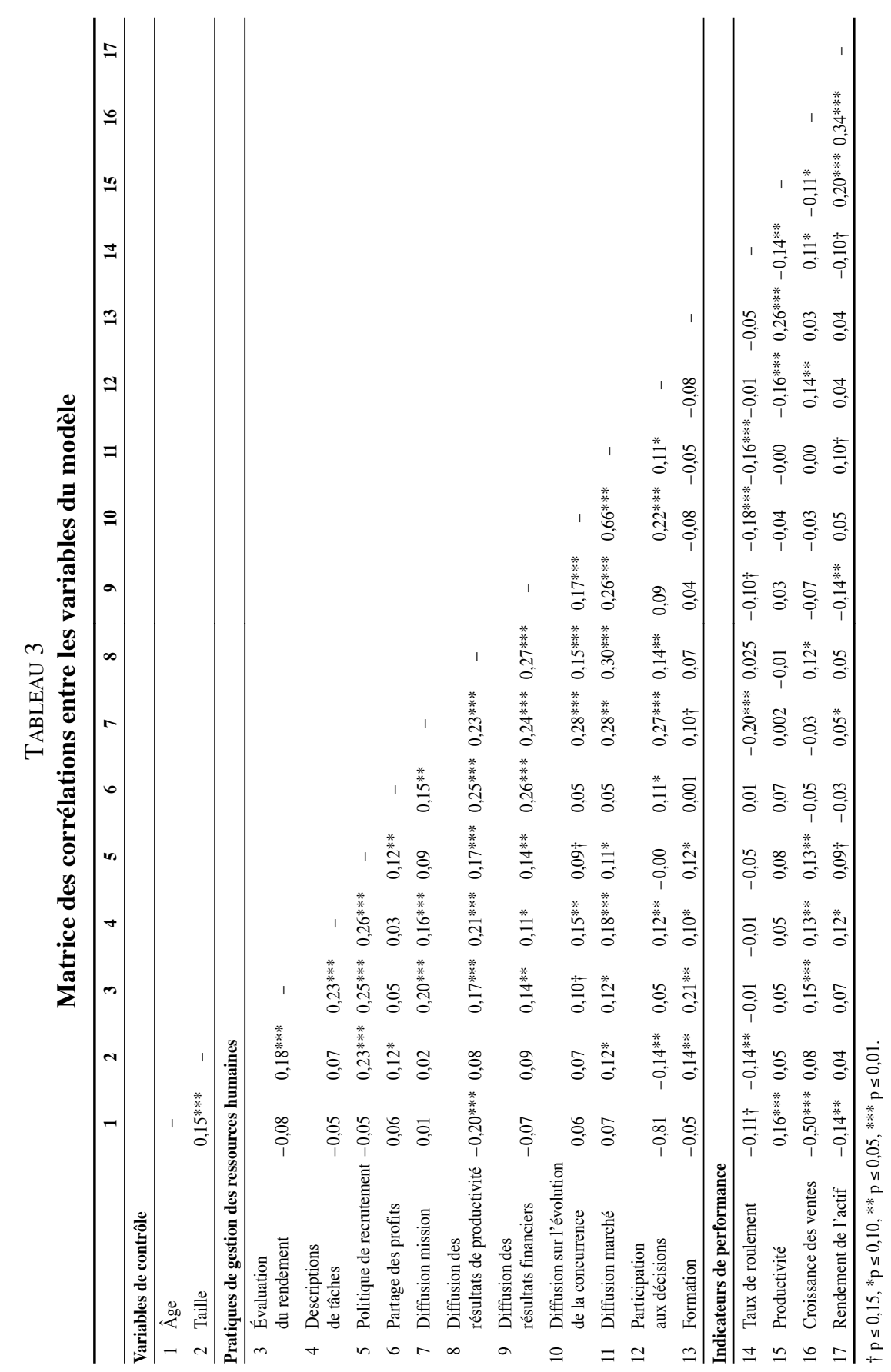

Revue internationale P.M.E., vol. 18, $\mathrm{n}^{\mathrm{o}} 2,2005$ 
rapport aux moyennes. Les sous-échantillons ainsi obtenus regroupent, pour l'un, les entreprises dont le nombre d'employés est inférieur à 48 (E2) et, pour l'autre, les entreprises dont le nombre d'employés est supérieur à 48 (E3). Précisons que pour effectuer nos analyses multivariées, certaines variables dépendantes ont dû subir des transformations visant à assurer leur conformité avec les hypothèses de régression linéaire.

Le tableau 4 illustre les résultats obtenus en ce qui concerne les impacts des pratiques de GRH sur les différents indicateurs de performance, selon que nous avons affaire à l'échantillon complet (E1), au groupe d'entreprises de moins de 48 employés (E2) ou au groupe d'entreprises de plus de 48 employés (E3).

\subsubsection{Variance observée}

Avant d'examiner la contribution de l'une ou l'autre des variables contenues dans les différents modèles, observons tout d'abord que la part de variance expliquée varie considérablement selon l'indicateur de performance pris en compte. Ainsi, les modèles relatifs au taux de roulement du personnel et au taux de roulement de l'actif n'arrivent à expliquer que des parts relativement faibles de variance, oscillant entre $3 \%$ et $7 \%$. Les modèles liés à la productivité expliquent pour leur part de $13 \%$ à $18 \%$ de la variance observée. Quant aux modèles relatifs à la croissance moyenne des ventes, ils arrivent à expliquer des parts de variance allant de $27 \%$ à $41 \%$. Notons par ailleurs que, comme le veut le type d'analyse multivariée que nous avons retenu, tous les modèles présentés au tableau 4 sont statistiquement significatifs au seuil habituel de $10 \%$.

\subsubsection{Variables de contrôle}

\section{Âge}

L'âge des entreprises ressort comme une variable qui contribue fortement à l'explication de la variance observée dans les indicateurs de performance que sont la productivité, la croissance des ventes et le taux de rendement de l'actif. L'âge présente en effet une relation significative avec la productivité des PME, tant dans l'échantillon complet $(\beta=0,17 ; \mathrm{p} \leq 0,01)$ que dans le sous-échantillon des plus petites PME $(\beta=0,17 ; p \leq 0,1)$. La relation entre l'âge et la productivité n'est cependant plus significative lorsqu'on ne considère que les plus grandes PME $(\beta=0,11 ; p>0,1)$. L'âge se révèle également une variable fortement significative si l'on considère la croissance des ventes. En fait, le taux de croissance des ventes diminue avec l'âge, et ce, tant dans l'échantillon complet $(\beta=-0,51 ; \mathrm{p} \leq 0,01)$ que dans les sous-échantillons des plus petites $(\beta=-0,49 ; \mathrm{p} \leq 0,01)$ et des plus grandes $(\beta=-0,54 ; \mathrm{p} \leq 0,01)$ PME. Enfin, on observe que l'âge présente une 


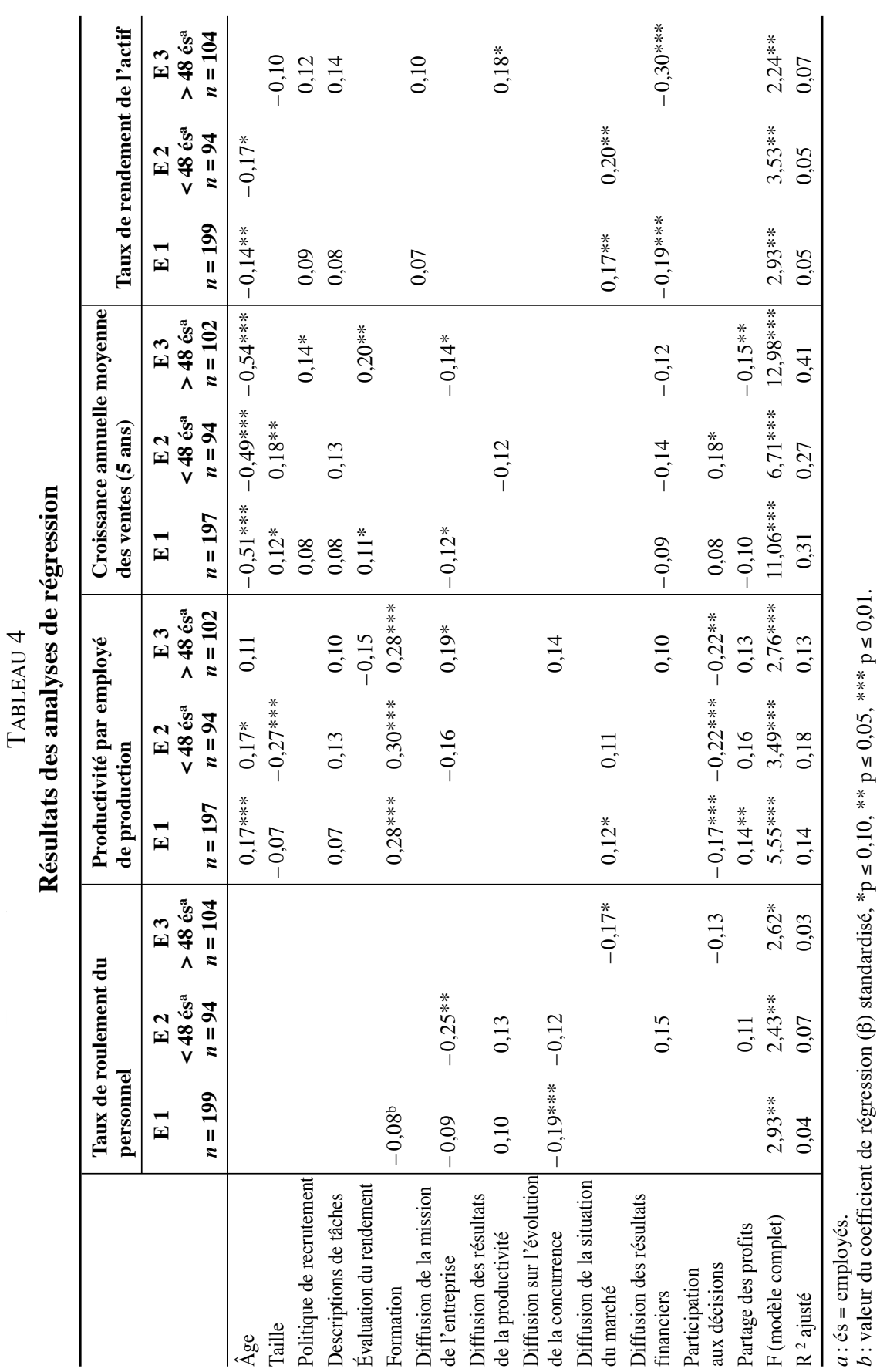

Revue internationale P.M.E., vol. 18, nº 2, 2005 
relation négative et significative avec le taux de rendement de l'actif, à la fois dans l'échantillon complet $(\beta=-0,14 ; \mathrm{p} \leq 0,05)$ et dans le sous-échantillon des plus petites PME $(\beta=-0,17 ; \mathrm{p} \leq 0,1)$.

Les coefficients négatifs obtenus pour la croissance moyenne des ventes et le taux de rendement de l'actif peuvent s'expliquer par le fait que les entreprises plus âgées ont atteint leur vitesse de croisière et que le taux de croissance des ventes, tout comme le taux de rendement de l'actif, y est moins spectaculaire que dans les jeunes entreprises. En revanche, les entreprises plus âgées affichent une meilleure productivité, ce qui peut s'expliquer par une plus grande expérience et un meilleur contrôle des procédés de production.

Taille

La taille des entreprises présente des corrélations significatives avec la productivité des employés de production et la croissance moyenne des ventes. L'effet de la taille sur ces deux indicateurs prévaut surtout dans les PME de moins de 48 employés (E2), aucune relation significative n'étant observée dans les PME de plus de 48 employés (E3). Dans le cas de la productivité, la relation observée revêt un signe négatif $(\beta=-0,27 ; p \leq 0,01)$. En ce qui concerne la croissance des ventes, par contre, l'effet de la taille se révèle positif $(\beta=0,18 ; \mathrm{p} \leq 0,05)$.

\subsubsection{Variables indépendantes}

\section{Recrutement}

La politique de recrutement est associée de façon significative $(\beta=0,14 ; \mathrm{p} \leq 0,1)$ à la croissance des ventes des PME comptant plus de 48 employés (E3). Cette pratique n'est associée de façon significative à aucun autre indicateur de performance.

\section{Descriptions de tâches}

Bien qu'elle soit retenue dans plusieurs modèles comme l'une des variables pouvant expliquer la variance observée dans les indicateurs de performance, la contribution de la pratique de descriptions de tâches n'apparaît pas significative d'un point de vue statistique.

\section{Évaluation du rendement}

L'évaluation du rendement ressort comme l'une des variables qui contribue de façon significative $(\beta=0,20 ; \mathrm{p} \leq 0,05)$ à l'explication de la croissance des ventes des PME de plus de 48 employés (E3). L'effet demeure significatif $(\beta=0,11$; $\mathrm{p} \leq 0,1)$, bien qu'atténué, dans l'échantillon complet (E1) et il s'évanouit dans les plus petites PME (E2). 


\section{Formation}

De toutes les pratiques de GRH, la formation est celle dont la contribution, observable essentiellement dans la productivité des employés, se révèle la plus significative, et ce, tant au sein de l'échantillon complet $(\beta=0,28 ; \mathrm{p} \leq 0,01)$ que dans les échantillons des plus petites $(\beta=0,30 ; \mathrm{p} \leq 0,01)$ et des plus grandes $(\beta=0,28$; $\mathrm{p} \leq 0,01)$ PME. De concert avec les autres variables retenues, la formation contribue à expliquer de $13 \%$ à $18 \%$ de la variance observée dans la productivité des entreprises.

\section{Diffusion d'information}

La pratique de diffusion d'information donne des résultats souvent positifs, mais parfois négatifs, suivant le type d'information diffusée et l'indicateur de performance pris en considération. En ce qui concerne les effets positifs et statistiquement significatifs tout d'abord, la diffusion d'information contribuerait à diminuer le taux de roulement du personnel au sein de l'échantillon E1 (évolution de la concurrence: $\beta=-0,19 ; \mathrm{p} \leq 0,01$ ), de même qu'au sein des échantillons E2 (mission d'entreprise: $\beta=-0,25$; $\mathrm{p} \leq 0,05$ ) et $\mathrm{E} 3$ (situation du marché : $\beta=-0,17$; $\mathrm{p} \leq$ $0,1)$. La diffusion d'information contribuerait également à expliquer l'amélioration de la productivité dans les échantillons $\mathrm{E} 1$ (situation du marché: $\beta=0,12 ; \mathrm{p} \leq 0,1$ ) et E3 (mission d'entreprise: $\beta=0,19 ; \mathrm{p} \leq 0,1$ ). Sauf lorsqu'elle porte sur les résultats financiers, la diffusion d'information exercerait en outre des effets positifs sur le taux de rendement de l'actif, lesquels effets sont observables en E1 (situation du marché: $\beta=0,17 ; p \leq 0,05)$, E2 (situation du marché : $\beta=0,20 ; p \leq 0,05$ ) et E3 (résultats de productivité: $\beta=0,18 ; \mathrm{p} \leq 0,1)$. Quant aux effets négatifs et statistiquement significatifs de la diffusion d'information, on les observe principalement dans la croissance des ventes, dans les échantillons $\mathrm{E} 1$ (mission d'entreprise : $\beta=-0,12$; $\mathrm{p} \leq 0,1)$ et $\mathrm{E} 3$ (mission d'entreprise : $\beta=-0,14 ; \mathrm{p} \leq 0,1$ ), de même que dans le taux de rendement de l'actif, à la fois dans l'échantillon complet (résultats financiers : $\beta=-0,19 ; \mathrm{p} \leq 0,01$ ) et dans celui des plus grandes PME (résultats financiers : $\beta=-0,30 ; p \leq 0,01)$.

\section{Participation aux décisions}

La participation aux décisions aurait des effets négatifs et significatifs sur la productivité des employés de production, tant au sein de l'échantillon complet $(\beta=-0,17$; $\mathrm{p} \leq 0,01)$ que dans celui des plus petites $(\beta=-0,22 ; \mathrm{p} \leq 0,01)$ et des plus grandes $(\beta=-0,22 ; p \leq 0,05)$ PME. En revanche, la participation aux décisions favoriserait la croissance des ventes des plus petites $\operatorname{PME}(\beta=0,18 ; \mathrm{p} \leq 0,1)$.

Revue internationale P.M.E., vol. 18, n 2, 2005

(c) 2005 - Presses de l'Université du Québec

Édifice Le Delta I, 2875, boul. Laurier, bureau 450, Sainte-Foy, Québec G1V 2M2 • Tél.: (418) 657-4399 - www.puq.ca

Tiré de: Revue internationale P.M.E., vol. 18, $\mathrm{n}^{\circ} 2$, sous la direction de Louis Raymond • PME1802N

Tous droits de reproduction, de traduction et d'adaptation réservés 


\section{Partage des profits}

Enfin, la pratique de partage des profits exercerait un effet positif et significatif sur la productivité des employés, comme on peut l'observer dans l'échantillon $\mathrm{E} 1(\beta=0,14 ; p \leq 0,05)$. Cette même pratique aurait toutefois un effet négatif et significatif sur la croissance des ventes des plus grandes PME $(\beta=-0,15 ; p \leq 0,05)$.

\section{Discussion}

À notre question de recherche visant à savoir si l'étendue d'application de certaines pratiques de GRH peut influencer la performance des PME nous pouvons répondre par l'affirmative. Les résultats obtenus avec les analyses de corrélation d'abord, puis avec les analyses multivariées, supportent l'hypothèse universaliste voulant que les pratiques les plus reconnues de gestion des ressources humaines soient associées à une meilleure performance des entreprises. À l'exception des descriptions de tâches et de la diffusion d'information relative aux résultats financiers, chacune des pratiques étudiées présente, dans l'un ou l'autre des modèles, au moins une relation significative allant dans le sens d'une amélioration de la performance des PME. Le plus souvent, les relations significatives vont dans le sens attendu. Certaines relations négatives, inattendues au départ, contribuent par ailleurs à nuancer un peu l'approche universaliste, comme nous en discuterons plus loin.

Globalement, nos résultats vont dans le même sens que ceux obtenus par d'autres chercheurs quant aux effets positifs que pourraient exercer, sur la performance d'entreprises, les pratiques de recrutement (Patterson et al., 1998; Way, 2002), d'évaluation du rendement (Guzzo, Jette et Katzell, 1985 ; Patterson et al., 1998; Arcand, Bayad et Fabi, 2002), de diffusion d'information (Arcand, Bayad et Fabi, 2002), de formation (Guzzo, Jette et Katzell, 1985 ; Patterson et al., 1998) et de partage des profits (Arcand, Bayad et Fabi, 2002; Way, 2002). À certains égards, nos résultats divergent cependant de ceux de Arcand et al. (2002), qui n'ont relevé aucun impact pour les pratiques de recrutement et de formation, de même que de ceux de Patterson et al. (1998), qui n'ont constaté aucun impact pour les pratiques de rémunération incitative et de diffusion d'information. Nos résultats témoignant d'une relation négative entre la participation aux décisions et la productivité contredisent également les résultats obtenus par Patterson et al. (1998). Notons par ailleurs que la convergence ou la divergence des résultats doit être nuancée étant donné que les variables étudiées ne sont pas mesurées de la même façon. 
L'un des premiers constats découlant de nos résultats réside dans les effets positifs spécifiques pouvant être attribués aux différentes pratiques. On observe ainsi que l'effet des pratiques de recrutement et d'évaluation du rendement se fait sentir principalement sur la croissance des ventes; celui de la formation, principalement sur la productivité; celui de la participation aux décisions, sur la croissance des ventes et celui du partage des profits, sur la productivité. L'effet positif de la diffusion d'information (prise au sens large) est le plus généralisé: on l'observe à la fois sur le taux de roulement du personnel, sur la productivité et sur le taux de rendement de l'actif. Il ressort clairement, par ailleurs, que certaines pratiques pourraient être contre-indiquées dans certaines situations. C'est le cas, notamment, de la participation aux décisions, qui irait à l'encontre d'une amélioration de la productivité, et de la diffusion d'information portant sur les résultats financiers, qui présente une forte relation négative avec le taux de rendement de l'actif.

On note également que trois pratiques de GRH (formation, diffusion d'information et partage des profits) seraient susceptibles d'affecter positivement la productivité des employés et que trois autres (recrutement, évaluation du rendement et participation aux décisions) pourraient affecter la croissance des ventes, alors qu'une seule pratique, soit la diffusion d'information (sous différentes formes) exercerait des effets positifs sur le taux de roulement du personnel et sur le taux de rendement de l'actif. De la même manière, on arrive à expliquer davantage de variance dans le cas de la productivité (jusqu'à 18\%) et de la croissance des ventes (jusqu'à $41 \%$ ) que dans le cas du taux de roulement du personnel (jusqu'à $7 \%$ ) et du taux de rendement de l'actif (jusqu'à 7\%). Il ressort de ce qui précède que l'effet des pratiques de GRH est plus perceptible au regard de la productivité et de la croissance des ventes; le lien entre les pratiques de GRH et ces deux indicateurs de performance pourrait donc être plus direct qu'il ne l'est pour les deux autres (taux de roulement du personnel et taux de rendement de l'actif).

Les relations négatives observées entre certaines pratiques de GRH et certains indicateurs de performance méritent par ailleurs qu'on s'y arrête. Ainsi, on peut s'étonner du fait que les PME où la productivité est plus forte soient également celles où l'étendue de la pratique de participation aux décisions est moindre, et ce, quelle que soit leur taille. À cet égard, la divergence entre nos résultats et ceux obtenus dans d'autres études (Patterson et al., 1998) est peut-être attribuable à notre méthode de mesure, qui tient compte de l'étendue des pratiques plutôt que de leur simple présence. Cependant, nos résultats nous autorisent à penser qu'une forte étendue de la participation aux décisions peut favoriser la croissance des ventes, comme le montrent les résultats obtenus pour cet indicateur de performance dans le sous-échantillon des plus petites entreprises. Selon que l'amélioration de la productivité constitue ou non une priorité pour l'entreprise (au détriment, par exemple, de la croissance ou de l'innovation), la participation aux

Revue internationale P.M.E., vol. 18, n 2, 2005

(C) 2005 - Presses de l'Université du Québec

Édifice Le Delta I, 2875, boul. Laurier, bureau 450, Sainte-Foy, Québec G1V 2M2 • Tél.: (418) 657-4399 - www.puq.ca

Tiré de: Revue internationale P.M.E., vol. 18, n², sous la direction de Louis Raymond • PME1802N

Tous droits de reproduction, de traduction et d'adaptation réservés 
décisions pourrait donc être une pratique plus ou moins pertinente. Nous atteignons en quelque sorte ici les limites de la perspective universaliste de la GRH. Le fait de présumer une interaction possible entre les priorités stratégiques de l'entreprise et les pratiques de GRH nous mène tout droit à l'approche de contingence, qui ne relève pas de notre propos dans le cadre du présent article.

On pourrait également s'étonner du fait que les pratiques de partage des profits et de diffusion d'information (mission de l'entreprise) soient associées de façon négative à la croissance des ventes. Ces mêmes pratiques, toutefois, sont associées positivement à la productivité, ce qui laisse croire, ici encore, à la pertinence de prendre en compte certains facteurs contextuels lorsque vient le temps de mettre en place les pratiques de GRH.

Quant à la valeur négative du coefficient de régression relatif à la diffusion des résultats financiers, une explication possible serait que les propriétaires jouissant des meilleurs rendements préfèrent user de prudence face à des employés qui pourraient réclamer une part plus importante des gains.

\subsection{Retombées}

En matière de recherche, comme nous venons de le voir, les résultats de cette étude montrent que l'approche universaliste peut fournir un éclairage intéressant sur les relations potentielles entre diverses pratiques de GRH et la performance des PME. Malgré leur orientation stratégique, les PME semblent en mesure de tirer profit de l'application de certaines pratiques. Les bénéfices obtenus pourront toutefois varier selon les indicateurs de performance observés et la taille des entreprises.

En ce qui concerne les implications de gestion, notre étude fournit aux dirigeants de PME des indications précieuses sur les différentes pratiques de GRH et leurs impacts potentiels. Selon qu'ils souhaitent favoriser l'un ou l'autre des indicateurs de performance de leur entreprise, les gestionnaires pourront insister davantage sur l'une ou l'autre des pratiques. Nos résultats soulignent en outre la nécessité de prendre en considération les objectifs prioritaires de l'entreprise avant d'appliquer certaines pratiques telles que la participation aux décisions, le partage des profits et la diffusion des résultats financiers, dont les effets peuvent être tantôt favorables, tantôt défavorables, selon les priorités établies. Les dirigeants de PME ne disposant pas à l'interne d'une expertise suffisante auraient en ce sens tout avantage à faire appel à une expertise externe pour les assister dans le choix et la mise en place des pratiques appropriées.

Revue internationale P.M.E., vol. 18, nº 2, 2005

(C) 2005 - Presses de l'Université du Québec

Édifice Le Delta I, 2875, boul. Laurier, bureau 450, Sainte-Foy, Québec G1V 2M2 • Tél.: (418) 657-4399 - www.puq.ca

Tiré de: Revue internationale P.M.E., vol. 18, $\mathrm{n}^{\circ} 2$, sous la direction de Louis Raymond PME1802N

Tous droits de reproduction, de traduction et d'adaptation réservés 
Il ressort de nos résultats que la diffusion d'information au plus grand nombre possible d'employés pourrait contribuer de façon significative à réduire le taux de roulement du personnel. Les sujets d'information à privilégier pourront varier selon la taille des entreprises, les plus petites ayant intérêt à insister sur la mission et les plus grandes, sur la situation du marché.

Par ailleurs, nos résultats indiquent clairement que la formation des employés contribuerait de façon substantielle à l'amélioration de la productivité des employés de production. L'impact de la formation sur la productivité est en fait le plus important que l'on puisse observer en matière de pratique de GRH dans l'ensemble des modèles analysés précédemment. Qui plus est, cet impact se vérifie dans chacun des échantillons, bien que la variance expliquée dans l'augmentation de la productivité atteigne son maximum (18\%) dans le sous-échantillon des plus petites PME. Après la formation, qui les précède de loin, les pratiques de partage des profits et de diffusion d'information (situation du marché) sembleraient les plus indiquées pour les PME recherchant une amélioration de la productivité.

Pour l'ensemble des PME, la pratique d'évaluation du rendement est associée positivement à la croissance moyenne des ventes. Les pratiques de recrutement, surtout dans les plus grandes PME et de participation aux décisions, surtout dans les plus petites PME, sont également associées à un accroissement des ventes.

Enfin, la diffusion d'information portant sur les résultats de productivité entraînerait un effet positif sur le taux de rendement de l'actif de l'ensemble des PME, mais plus encore au sein des petites, tandis que la diffusion d'information portant sur les résultats de productivité conviendrait aux plus grandes PME.

\section{Limites et conclusion}

Cette étude avait pour but de vérifier l'impact de certaines pratiques de GRH sur la performance de PME. Nous avons exposé, dans notre cadre théorique, différentes approches sur lesquelles on s'appuie généralement pour expliquer en quoi certaines pratiques de GRH pourraient affecter la performance des entreprises. Notre hypothèse est supportée par les résultats obtenus, plusieurs des pratiques étudiées apparaissant clairement associées à une amélioration de la performance des PME. Nos résultats permettent, croyons-nous, de mieux comprendre les impacts potentiels des différentes pratiques lorsqu'elles sont appliquées en contexte de PME, tout comme ils permettent de nuancer l'approche universaliste de la GRH. Il semble en fait que ce ne soient pas toutes les entreprises qui bénéficient de l'application intensive de certaines des pratiques de GRH figurant pourtant parmi les plus

Revue internationale P.M.E., vol. 18, n 2, 2005

(C) 2005 - Presses de l'Université du Québec

Édifice Le Delta I, 2875, boul. Laurier, bureau 450, Sainte-Foy, Québec G1V 2M2 • Tél.: (418) 657-4399 - www.puq.ca

Tiré de: Revue internationale P.M.E., vol. 18, n², sous la direction de Louis Raymond • PME1802N

Tous droits de reproduction, de traduction et d'adaptation réservés 
«stratégiques». Comme le montrent nos résultats, la taille semble être un facteur non négligeable lorsqu'on veut étudier et mieux comprendre les modalités d'application des pratiques de GRH dans les PME.

Nos résultats révèlent également que la définition de la mesure de la performance est critique pour l'évaluation de la pertinence ou de l'effet de l'implantation de certaines pratiques. En effet, les mêmes pratiques n'influencent pas de façon uniforme tous les indicateurs de performance. Les différences observées entre le niveau des coefficients de régression obtenus selon les différents indicateurs témoignent de l'importance d'une définition rigoureuse de ce concept.

Toute recherche comporte ses limites et la nôtre n'échappe pas à cette règle, d'autant plus que la gestion des ressources humaines en contexte de PME représente un domaine de recherche très jeune et qui n'en est encore qu'au stade exploratoire. Le modèle issu de la perspective universaliste de la GRH revêt, il faut bien le dire, un caractère un peu simpliste, en ce sens qu'il ignore les différentes variables de contingence qui pourraient interagir avec les variables dépendantes. Les indicateurs de performance, s'ils peuvent être influencés par des facteurs internes, n'en sont pas moins sujets à varier également en fonction des réalités économiques et des bouleversements de l'environnement externe, tout comme ils peuvent fluctuer en fonction de certaines caractéristiques qui confèrent aux PME le caractère d'hétérogénéité qu'on leur connaît. Or, ces différents facteurs n'ont pas été pris en considération dans la présente étude.

Il convient de rappeler, par ailleurs, que les résultats obtenus l'ont été à partir d'une étude en coupe instantanée et qu'ils ne peuvent donner lieu à aucun lien de causalité quant au sens des relations identifiées. La fameuse question de savoir si les entreprises affichent une meilleure performance parce qu'elles appliquent davantage de pratiques de GRH ou si elles appliquent davantage de pratiques parce qu'elles sont plus performantes demeure entière. Rappelons également que notre échantillon est constitué de PME ayant choisi d'adhérer volontairement à une activité d'étalonnage, ce qui reflète une certaine préoccupation pour l'évaluation de leur performance. Une telle situation limite évidemment les possibilités de généralisation en dehors de l'univers restreint et non probabiliste que constitue notre échantillon.

En dépit de ces limites, notre étude ouvre tout de même la voie à d'autres recherches. En premier lieu, il nous semblerait important d'améliorer la compréhension du comportement des PME en matière de GRH en examinant, par exemple, jusqu'à quel point certaines variables de contingence (profil du propriétaire, innovation, technologies, exportation, etc.) agissent comme déterminants de l'adoption des différentes pratiques. Il nous apparaîtrait pertinent, par ailleurs, de vérifier l'interaction possible entre la stratégie d'affaires adoptée par les PME, la présence d'une expertise en GRH, les pratiques (ou ensembles de pratiques) mises 
en place et la performance obtenue à divers égards. Enfin, il nous semblerait approprié de recourir à des méthodes statistiques telle l'équation structurelle afin de vérifier le rôle intermédiaire que pourraient jouer certains indicateurs de performance (par exemple la productivité et la croissance des ventes) lorsque vient le temps de mesurer l'impact des pratiques de GRH sur la performance financière (par exemple le taux de rendement de l'actif) des PME.

Souhaitons, en terminant, que notre recherche ait non seulement contribué à une meilleure compréhension des impacts de la GRH sur la performance des PME, mais qu'elle ait aussi pour effet de stimuler la recherche visant à développer un modèle qui convienne bien à l'analyse des pratiques de gestion des ressources humaines dans le contexte particulier de ces entreprises.

\section{Bibliographie}

ArCAND, M., M. BAYAD et B. FABI (2002), «L'effet des pratiques de gestion des ressources humaines sur l'efficacité organisationnelle des coopératives financières canadiennes », Annals of Public and Cooperative Economics, vol. 73, $\mathrm{n}^{\circ}$ 2, p. 215-240.

AhMAD, S. ET R.G. SCHROEDER (2003), «The impact of human resource management practices on operational performance: recognizing country and industry differences», Journal of Operations Management, vol. 21, n 1, p. 19-43.

BARNEY, J. (1991), «Firm resources and sustained competitive advantage», Journal of Management, vol. 17, n 1, p. 99-120.

BARNEY, J. et P.M. WRIGHT (1998), «On becoming a strategic partner: the role of human resources in gaining competitive advantage», Human Resource Management, vol. $37, \mathrm{n}^{\mathrm{o}} 1$, p. 31-46.

BECKER, G. (1962), «Investment in human capital: a theoretical analysis », Journal of Political Economy, vol. 70, octobre, p. 9-49.

BECKER, B. et B. GERHART (1996), «The impact of human resource management on organizational performance: progress and prospects », Academy of Management Journal, vol. 39, no 4, p. 779-801.

BECKER, B.E. et M.A. HusELID (1998), «High performance work systems and firm performance: a synthesis of research and managerial implications », Research in Personnel and Human Resources Management, vol. 16, p. 53-101.

Becker, B.E., M.A. Huselid, P.S. Pickus et M.F. SPRatT (1997), «HR as a source of shareholder value research and recommendations », Human Resource Management, vol. 36, no 1, p. 39-47.

Bowen, D.E., S.W. GiLliland et R. Folger (1999), «HRM and service fairness: how being fair with employees spills over to customers», Organizational Dynamics, vol. 27, hiver, p. 7-23.

Revue internationale P.M.E., vol. 18, nº 2, 2005

(C) 2005 - Presses de l'Université du Québec

Édifice Le Delta I, 2875, boul. Laurier, bureau 450, Sainte-Foy, Québec G1V 2M2 - Tél.: (418) 657-4399 - www.puq.ca

Tiré de: Revue internationale P.M.E., vol. 18, n² 2 , sous la direction de Louis Raymond P PME1802N

Tous droits de reproduction, de traduction et d'adaptation réservés 
Bryson, A. (1999), «The impact of employee involvement on small firms' financial performance», National Institute Economic Review, juillet, no 169, p. 78-95.

CAPelli, P. et A. CRocker-Hefter (1996), «Distinctive human resources are firm's core competencies », Organizational Dynamics, vol. 24, n 3, p. 7-22.

COLBERT, B.A. (2004), «The complex resource-based view: implications for theory and practice in strategic human resource management», Academy of Management Review, vol. 29, no 3, p. 341-358.

D’Amboise, G. et D.J. Garand (1995), «Identification des difficultés et besoins des PME en matière de gestion des ressources humaines », Gestion 2000, vol. 11, n 1 , p. 109-132.

D'Arcimoles, C.H. (1995), Diagnostic financier et gestion des ressources humaines: nécessité et pertinence du bilan social, Paris, Economica.

DELANEY, J. et M.A. HuSELID (1996), «The impact of human resource management practices on perceptions of organizational performance», Academy of Management Journal, vol. 39, nº 4, p. 949-969.

DELERY, J.E. et D.H. Doty (1996), «Modes of theorizing in strategic human resource management: tests of universalistic, contingency and configurational performance predictions », Academy of Management Journal, vol. 39, no 4, p. 802-835.

Doty, D.H., W.H. GLICK et G.P. Huber (1993), «Fit, equifinality, and organizational effectiveness: a test of two configurational theories », Academy of Management Journal, vol. 36, nº 6, p. 1196-1250.

Ducharme, L.M. (1998), «Introduction: main theories and concepts», dans Measuring Intangible Investment, Paris, OCDE.

FABI, B. et D.J. GARAND (à paraître), «La gestion des ressources humaines », dans PierreAndré Julien (dir.), Les PME, bilan et perspectives, 3 e édition, Cap-Rouge, Presses Interuniversitaires.

FABI, B., Y. MARTIN et P. VALOIS (1999), «Favoriser l'engagement organisationnel des personnes œuvrant dans une organisation en transformation », Gestion, vol. 24, n 3 , p. 102-113.

GuZzo, R.A., R.D. JeTtE et R.A. KATZELL (1985), «The effects of psychologically based intervention programs on worker productivity: a meta analysis », Personnel Psycho$\log y$, vol. $38, \mathrm{n}^{\circ} 2$, p. 275-291.

HANSEN, G.S. et B. WERNERFELT (1989), «Determinants of firm performance: the relative importance of economic and organizational factors», Strategic Management Journal, vol. 10, no 5, p. 399-411.

HoRnSBY, J.S. et D.F. KURATKO (1990), «Human resource management in small business: critical issues for the 1990's », Journal of Small Business Management, vol. 8, no 3, p. 9-18.

HoRnSBY, J.S. et D.F. KURATKO (2003), «Human resource management in U.S. small businesses: a replication and extension», Journal of Developmental Entrepreneurship, vol. 8, n$^{\circ} 1$, p. $73-92$.

Revue internationale P.M.E., vol. 18, nº 2, 2005

(C) 2005 - Presses de l'Université du Québec

Édifice Le Delta I, 2875, boul. Laurier, bureau 450, Sainte-Foy, Québec G1V 2M2 • Tél.: (418) 657-4399 - www.puq.ca

Tiré de: Revue internationale P.M.E., vol. 18, n² 2, sous la direction de Louis Raymond • PME1802N

Tous droits de reproduction, de traduction et d'adaptation réservés 
HuSELID, M.A. (1995), «The impact of human resource management practices on turnover, productivity, and corporate financial performance», Academy of Management Journal, vol. 38, no 3, p. 635-672.

Huselid, M.A., S.E. JACKSON et R.S. SCHUlER (1997), «Technical and strategic human resource management effectiveness as determinants of firm performance», Academy of Management Journal, vol. 40, nº 1, p. 171-188.

JACKSON, S.E. et R.S. SCHULER (1995), «Understanding human resource management context of organizations and their environments», dans M.R. Rosenzweig et L.W. Porter (dir.), Annual Review of Psychology, vol. 46, nº 1, p. 237-264.

JEnSEn, M. et W. Meckling (1976), «Theory of the firm: managerial behavior, agency costs, and ownership structure», Journal of Financial Economics, vol. 3, n ${ }^{\circ} 4$, p. $305-360$.

JoNES, G.R. et P.M. WRIGHT (1992), «An economic approach to conceptualizing the utility of human resource management practices », Research in Personnel and Human Resources Management, vol. 10, p. 271-299.

KaPlan, R.S. et D.P. NoRTON (1992), «The balanced scorecard: measures that drive performance», Harvard Business Review, vol. 70, n 1, janvier-février, p. 71-79.

Katz, J.A., H.E. Aldrich, T.M. Welbourne et P.M. Williams (2000), «Guest editor's comments. Special issue on human resource management and the SME: toward a new synthesis », Entrepreneurship Theory and Practice, vol. 25, nº 1, p. 7-10.

KenNERley, M. et A. NeEly (2003), «Measuring performance in a changing business environment», International Journal of Operations \& Production Management, vol. 23, no 2, p. 213-229.

KerR, A. et M. MCDougall (1999), «The small business of developing people», International Small Business Journal, vol. 17, $\mathrm{n}^{\circ}$ 2, p. 65-74.

LACOURSIÈRE, R. (2002), «Impacts de certaines pratiques stratégiques de gestion des ressources humaines sur la performance organisationnelle et financière de PME», Mémoire de maîtrise, Université du Québec à Trois-Rivières.

LEPAK, D.P. et S.A. SNELl (1999), «The human resource architecture: toward a theory of human capital allocation and development», Academy of Management Review, vol. 24, no 1, p. 31-48.

Liouville, J. et M. BAYAD (1995), «Stratégies de gestion des ressources humaines et performances dans les PME: résultats d'une recherche exploratoire », Gestion 2000, vol. 1, p. 159-179.

McMahan, G., M. VIRIK et P.M. Wright (1999), «Theoretical perspectives for SHRM», Research in Personnel and Human Resources Management, Supplément 4, p. 99-122.

Marlow, S. et D. PatTon (1993), «Managing the employment relationship in the smaller firm: possibilities for human resource management», note de recherche, International Small Business Journal, vol. 11, no 4, p. 57-64.

Revue internationale P.M.E., vol. 18, nº 2, 2005

(c) 2005 - Presses de l'Université du Québec

Édifice Le Delta I, 2875, boul. Laurier, bureau 450, Sainte-Foy, Québec G1V 2M2 • Tél.: (418) 657-4399 - www.puq.ca

Tiré de: Revue internationale P.M.E., vol. 18, n² 2 , sous la direction de Louis Raymond P PME1802N

Tous droits de reproduction, de traduction et d'adaptation réservés 
Meyer, A.D., A.S. Tsui et C.R. Hinings (1993), «Guest co-editor's introduction: configurational approaches to organizational analysis », Academy of Management Journal, vol. 36, no 6, p. 1175-1195.

NeEly, A., H. Richards, J. Mills, K. Platts et M. Bourne (1997), «Designing performance measures: a structured approach », International Journal of Operations \& Production Management, vol. 17, $\mathrm{n}^{\circ}$ 11, p. 1131-1152.

Patterson, M.G., M.A. West, R. Lawthom et S. Nickell (1998), «Impact of people management practices on business performance», Issues in People Management, $\mathrm{n}^{\circ}$ 22, Londres, Institute of Personnel and Development (IPD House).

PeterAf, M.A. (1993), «The cornerstones of competitive advantage: a resource-based view », Strategic Management Journal, vol. 14, n 3, p. 179-191.

Pfeffer, J. (1994), «Competitive advantage through people», California Management Review, vol. 36, n 2 , p. 9-28.

Prahalad, C.K. et G. HAmel (1990), «The core competence of the corporation », Harvard Business Review, vol. 68, n 3, p. 79-91.

PuRCELl, J. (1999), «Best practice and best fit: chimera or cul-de-sac?», Human Resource Management Journal, vol. 9, no 3, p. 26-41.

ST-PIERre, J. (1999), La gestion financière des PME: Théories et pratiques, Sainte-Foy, Presses de l'Université du Québec, coll. «Entrepreneuriat et PME».

Schultz, T.W. (1961), «Investment in human capital», American Economic Review, vol. $51, \mathrm{n}^{\circ} 1$, mars, p. 1-17.

SChumann, M. (1998), «New concepts of production and productivity», Economic and Industrial Democracy, vol. 19, $\mathrm{n}^{\circ}$ 1, p. 17-32.

TANSKY, J.W. et R. HenEman (2003), «Guest editor's note. Introduction to the special issue on human resource management in SMEs: a call for more research », Human Resource Management, vol. 42, n 4, p. 299-302.

TeEce, D.J., G. PisAno et A. ShuEn (1997), «Dynamic capabilities and strategic management», Strategic Management Journal, vol. 18, nº 7, p. 509-533.

Tetenbaum, T.J. (1998), «Shifting paradigms: from Newton to chaos», Organizational Dynamics, vol. 26, no 4, printemps, p. 21-32.

WAY, S.A. (2002), «High performance work systems and intermediate indicators of firm performance within the US small business sector», Journal of Management, vol. 28, no 6, p. 765-785.

WAY, S.A. et J.W. THACKER (2004), «Having a human resource manager in a Canadian small business: what difference does it make?», Journal of Small Business and Entrepreneurship, vol. 17, no 4, p. 293-300.

Welbourne, T.M. et L.A. Cyr (1999), «Using ownership as an incentive», Group and Organization Management, vol. 24, no 4, p. 438-460.

Wernerfelt, B. (1984), «A resource-based view of the firm», Strategic Management Journal, vol. 5, n 2 , p. 171-180. 
WiLLIAMSON, O.E. (1981), «The economics of organization: the transaction cost approach», American Journal of Sociology, vol. 87, n 3, p. 548-577.

Wright, P.M., B.B. Dunford et S.A. SNELl (2001), «Human resources and the resourcebased view of the firm », Journal of Management, vol. 27, $\mathrm{n}^{\circ}$ 6, p. 701-721.

Revue internationale P.M.E., vol. 18, $\mathrm{n}^{\circ}$ 2, 2005

(C) 2005 - Presses de l'Université du Québec

Édifice Le Delta I, 2875, boul. Laurier, bureau 450, Sainte-Foy, Québec G1V 2M2 • Tél.: (418) 657-4399 - www.puq.ca

Tiré de: Revue internationale P.M.E., vol. 18, n² , sous la direction de Louis Raymond P PME1802N

Tous droits de reproduction, de traduction et d'adaptation réservés 
(C) 2005 - Presses de l'Université du Québec

Édifice Le Delta I, 2875, boul. Laurier, bureau 450, Sainte-Foy, Québec G1V 2M2 • Tél.: (418) 657-4399 - www.puq.ca

Tiré de: Revue internationale P.M.E., vol. 18, $\mathrm{n}^{\circ}$ 2, sous la direction de Louis Raymond - PME1802N

Tous droits de reproduction, de traduction et d'adaptation réservés 